\title{
BMJ Open Equity in vaccine trials for higher weight people? Protocol for a rapid review of inclusion and exclusion criteria for higher weight people in clinical trials for COVID-19
}

To cite: Campbell J, Hobbs M, 0 'Hara L, et al. Equity in vaccine trials for higher weight people? Protocol for a rapid review of inclusion and exclusion criteria for higher weight people in clinical trials for COVID-19. BMJ Open 2021;11:e050114. doi:10.1136/ bmjopen-2021-050114

- Prepublication history and additional online supplemental material for this paper are available online. To view these files, please visit the journal online (http://dx.doi.org/10. 1136/bmjopen-2021-050114)

Received 10 February 2021 Revised 27 April 2021 Accepted 28 April 2021
Check for updates

(C) Author(s) (or their employer(s)) 2021. Re-use permitted under CC BY-NC. No commercial re-use. See rights and permissions. Published by BMJ.

For numbered affiliations see end of article.

Correspondence to

Ms Lesley Gray;

lesley.gray@otago.ac.nz

\section{ABSTRACT}

Introduction Vaccination is a public health strategy that aims to reduce the burden of viral illness, especially important for populations known or likely to be at increased risk for inequitable outcomes due to the disease itself or disparities in care accessed and received. The role of weight status in COVID-19 susceptibility and disease burden remains unclear. Despite this, higher weight is frequently described as a definitive risk factor for both susceptibility and disease severity. Therefore, COVID-19 vaccine trials should recruit a study group representative of the full weight spectrum, and undertake appropriate subgroup analysis by weight status to evaluate response and titrate dose regimes where indicated to ensure equitable outcomes for higher weight people.

Methods and analysis We aim to review inclusion and exclusion criteria of clinical trial protocols registered with ClinicalTrials.gov, ISRCTN Register, the WHO official vaccine trial register, and 'The COVID-19 Vaccine Tracker'. To determine the number of trials including higher weight (body mass index $>30 \mathrm{~kg} / \mathrm{m}^{2}$ ) individuals and the number of trials conducting efficacy subgroup analyses by weight status. Screening, data extraction and quality appraisal of trial protocols will be completed independently by a minimum of two reviewers. Clinical trials will be assessed for risk of bias using the Risk of Bias-2 tool. We will conduct a descriptive analysis of extracted data. The following subsets are proposed: participation of higher weight people in COVID-19 vaccine trials by trial phase, country and vaccine platform.

Ethics and dissemination Ethical approval was not required for this review. The results of this rapid review will be presented at appropriate conferences and published in a suitable peer reviewed journal.

PROSPERO registration number CRD42020226573

\section{INTRODUCTION}

The clinical presentation of Severe Acute Respiratory Syndrome Coronavirus 2 (SARS-CoV-2) infection ranges from asymptomatic infection to organ dysfunction presenting as shock, acute respiratory distress
Strengths and limitations of this study

- To date, no review has been conducted for the inclusion rates of higher weight people in COVID-19 vaccine trials.

- These findings will help inform potential limitations in the evidence currently available in formulating advice on COVID-19 vaccinations for higher weight people.

- The review method is based on the recent interim guidance from the Cochrane Rapid Reviews Methods Group.

- A minimum of two reviewers will independently screen registered protocols, extract data, appraise quality and conduct the analysis.

- The inclusion of studies published only in English may mean that important additional findings are missed.

syndrome, acute cardiac or kidney injury and death. ${ }^{12}$ A major clinical risk factor for COVID-19 is older age and other risk factors reported include male sex and chronic health conditions such as hypertension and cardiovascular disease. ${ }^{3} 4$ Groups reported with higher risk for severe COVID-19 include people with high body mass. ${ }^{5}$ There remains uncertainty about the role weight plays in COVID-19 susceptibility and disease burden.

Research and development of safe and effective SARS-CoV-2 vaccines began early in 2020 and there has been rapid movement from feasibility studies through to human clinical trials and administration of vaccines in several countries. ${ }^{6}$ The rapid rate of development has been credited to the large body of preclinical trial work previously undertaken in vaccine development for other similar coronaviruses such as Severe Acute Respiratory Syndrome Coronavirus 1 (SARS-CoV-1) 
and Middle Eastern Respiratory Syndrome Coronavirus 7 (MERS-CoV-7).

Vaccination is a public health strategy that aims to reduce the incidence and severity of viral illness and may also reduce community transmission. The new COVID-19 vaccines that have been approved for use in many countries have demonstrated high levels of efficacy in reducing the establishment of severe viral illness. The extent to which COVID-19 vaccines interrupt viral transmission is yet to be fully established, ${ }^{7}$ although reviews by national agencies such as the US Food and Drug Administration show high levels of short term safety and efficacy in protecting against symptomatic COVID-19 for a number of vaccines now approved for use in many countries. ${ }^{8}$ Many of the current trials in the development of an SARS-CoV-2 vaccine seek to induce neutralising antibodies (nAbs) to the spike protein on the surface of the virus. ${ }^{9}$ Induction of nAbs is associated with protection in previous viral vaccine development. ${ }^{9}$ Further to traditional inactivated viral vaccine candidates, a range of modern-as yet unlicensed technologies-are being evaluated against SARS-CoV-2. ${ }^{10}$

Historically, population groups such as elderly people, young children and pregnant women have been excluded from clinical trials based on their designation as vulnerable groups. ${ }^{11}{ }^{12}$ Higher weight people have also historically been excluded from clinical trials based on perceptions of vulnerability or in an attempt to reduce confounding. Such exclusions raise ethical and efficacy concerns. Exclusions of particular groups from clinical research can harm individuals by limiting their autonomy and denying them access to experimental interventions, and can harm groups by limiting the evidence available to inform their clinical care ${ }^{13}$ Lack of diverse representation reduces the generalisability of the research findings to the whole population and specific application to the population groups excluded. This can result in poorer quality care, higher rates of adverse events and worse health outcomes for groups excluded from clinical research. ${ }^{14}$ For example, WHO has indicated that the exclusion of pregnant women from COVID-19 vaccine research is a significant concern. ${ }^{15}$

There is some evidence of the impact of body weight on drug efficacy. Clinical trials of cancer therapeutics have come under scrutiny in recent years for the exclusion of higher weight participants. ${ }^{13}$ Vaccine efficacy (VE) across the weight spectrum has been unclear in prior vaccines. ${ }^{16}{ }^{17}$ An inverse relationship is reported between body mass index (BMI) and antibody response to a standard dose of HepA and HepB vaccination, with reduced antibody titres associated with higher weight status. ${ }^{16}$ Conversely in the administration of the trivalent inactivated influenza vaccine weight status was associated with higher initial antibody titres in participants of higher weight status, however by 12 months a greater decline in antibodies was noted. ${ }^{16}{ }^{17}$ Early evidence from three trials (Pfizer, Moderna and Johnson \& Johnson) shows that vaccine safety and efficacy is comparable in recipients across the weight spectrum. ${ }^{18} 19$
Ideally, COVID-19 vaccine trials should recruit a representative study group and undertake appropriate subgroup analysis by weight status, to evaluate safety, efficacy and titrate dose regimes where indicated to ensure equitable outcomes for higher weight people. ${ }^{1718}$ To date, no review has been conducted for the inclusion rates of higher weight people in COVID-19 vaccine trials.

\section{OVERALL OBJECTIVE}

The primary objective of this rapid review is to quantify the number of protocols that include higher weight people (defined as BMI of $30 \mathrm{~kg} / \mathrm{m}^{2}$ and above) as participants in clinical trials of novel vaccines for COVID-19. Secondary objectives are to quantify the number of higher weight people participating in these trials and quantify the number of phase 3 efficacy trials conducting analysis of $\mathrm{VE}$ across the weight spectrum.

\section{Specific review questions}

To conduct a rapid review of clinical trial protocols of COVID-19 novel vaccines in order to quantify:

1. The proportion of trials that include higher weight people, as determined by $\mathrm{BMI}>30 \mathrm{~kg} / \mathrm{m}^{2}$, as participants.

2. The proportion of higher weight individuals (BMI $>30$ $\mathrm{kg} / \mathrm{m}^{2}$ ) participating in novel COVID-19 vaccine trials.

3. The proportion of phase 3 trials conducting analysis of VE by weight status.

\section{METHODS}

\section{Study design}

A rapid review of registered vaccine trial protocols will be conducted with the aim of the review to be complete by 30 May 2021. The review method is based on the recent interim guidance from the Cochrane Rapid Reviews Methods Group. ${ }^{20}$ Rapid reviews have emerged as an efficient tool to get evidence to decision makers more quickly and are now considered part of the knowledge synthesis family. $^{21}$

Review constraints include:

1. A minimum of two reviewers will independently screen registered protocols, extract data, appraise quality and conduct the analysis.

2. Registered protocols in any stage prior to recruitment will be included in the primary objective analysis.

\section{Protocol registration}

Any revisions will be identified on the registered document. Post hoc changes will be reported with the published results. This protocol was drafted and written in accordance with the Preferred Reporting Items for Systematic Reviews and Meta-Analysis Protocols statement. ${ }^{22} 23$

\section{Eligibility criteria}

Protocols will be included in the review if they meet the following criteria. 
Types of studies

Inclusion criteria: trials of a novel COVID-19 vaccine in any clinical phase with registered protocols evaluating efficacy, safety and/or immunogenicity of the vaccine.

Exclusion criteria: clinical trials evaluating therapies that do not generate active immunity; trials including people infected by COVID-19; trials evaluating the efficacy of vaccines designed to protect against other pathogens eg, Bacille Calmette-Guerin (BCG) vaccine in the prevention or treatment of COVID-19; protocols written in a language other than English.

\section{Participants}

Any participant in the included studies with a negative test for COVID-19 infection at the time of recruitment. Participants will be adults, aged $\geq 18$ years, and included irrespective of sex, gender and ethnicity.

\section{Interventions(s) and exposure(s)}

Clinical trials in any phase for any novel COVID-19 vaccine, and participation in those trials with a focus on the participation of higher weight individuals.

\section{Comparator(s)/control}

Not applicable.

\section{Context/setting}

There are no restrictions on the setting, location or the country in which the trials may be registered and conducted.

\section{Outcome(s)}

The proportion of vaccine trials including higher weight individuals as participants as a proportion of total included COVID-19 vaccine trials.

The proportion of higher weight individuals participating in trials (where recruitment is complete) as a proportion of total included COVID-19 vaccine trial participants.

Of trials where recruitment is complete, the proportion of trials planning to stratify their VE estimates by weight status.

\section{Measures}

Total numbers and percentages.

\section{Time}

All periods of time and duration of follow-up.

\section{Search strategy}

A search will be performed of ClinicalTrials.gov, ISRCTN Register, the WHO official website for vaccine trials, 'The COVID-19 Vaccine Tracker' developed by the Vaccine Centre at the London School of Hygiene \& Tropical Medicine, and the Australia and New Zealand Clinical Trial Register to identify clinical trials for vaccines in different countries irrespective of publication status, publication year, and language ${ }^{24}{ }^{25}$ Further details of the search strategy are included in separate online supplemental table S1.
Data collection and appraisal

Data extraction (selection and coding)

Selection of trial protocols and data extraction will be performed by two reviewers (JC and LG) independently. Studies will be screened for relevance, and eligibility criteria as detailed above to be included in the review. Rayyan, a systematic review web-based application, will be used for selection and appraisal of protocols for eligibility with reviewer settings set to 'Blind ON'. ${ }^{26}{ }^{27}$ To reduce the risk of missing eligible protocols a low threshold for inclusion will be applied to screening decisions by two reviewers with a third reviewer checking excluded protocols $(\mathrm{MH})$. Disagreements will be resolved in consultation and by consensus (JC, LG and MH). A pilot review exercise will then be conducted independently by the review team on 10 selected trial protocols using a standardised data extraction form. Reviewers will examine any differences and calibrate the review form.

Each study will be reviewed using the calibrated data extraction form, collecting the following information. General study data: first author, year and language of publication, year and place(s) of study performance, trial number, trial status-recruiting, current, complete. Trial design: study phase, level of blinding, purpose- dose finding/dose confirmation. Participant characteristics: numbers, age range and mean (or median), health status. Vaccines: vaccine and placebo administered, antigen type/dose, adjuvant type/dose, vector type, dose regime. Risk of Bias (ROB): using the ROB-2 Tool, as described below (see online supplemental figure S1).

As outcomes, we will extract data on trials and review inclusion and exclusion criteria to determine participation of higher weight individuals, the number of higher weight individuals participating and whether the trial has, or will, conduct analysis of VE by weight status.

Where there is missing data or insufficient reporting in the protocol we will contact the protocol trial authors via email. Where communication is not received by 20th May, these will be reported as 'undefined'.

\section{Risk of Bias}

The risk of bias (ROB) in included studies will be independently evaluated by two reviewers (JC and LG). Any disagreements over ROB will be resolved in consultation with a third reviewer $(\mathrm{MH})$. To assess the risk of bias in controlled trials we will use the ROB-2 Cochrane risk of bias assessment tool. ${ }^{28} 29$

\section{Strategy for data synthesis}

We will conduct a descriptive analysis of extracted data to determine the total number of trials that include higher weight participants, the total number of higher weight people participating in trials and as a proportion of total participants across all trials. We will describe the number of trials evaluating the efficacy of the vaccine by weight status. We will present narrative and tabulated information for results. 


\section{Analysis of subgroups or subsets}

The following subsets are proposed: participation of higher weight people in COVID-19 vaccine trials by trial phase, country and vaccine platform.

\section{Ethics}

Ethical approval was not required for this review.

\section{DISSEMINATION}

Due to the urgency of disseminating evidence-based information, particularly as countries move rapidly to roll out vaccination programmes, we seek to undertake this review with similar urgency. Dissemination may include the use of medRxiv.org, for example, as 'preprints' of review findings prior to formal peer review. The results of this rapid review will be presented at appropriate conferences and will be published in a suitable peer-reviewed journal.

\section{Author affiliations}

${ }^{1}$ Otago Medical School, University of Otago, Christchurch, New Zealand

${ }^{2}$ College of Education, Health and Human Development, University of Canterbury, Christchurch, New Zealand

${ }^{3}$ Department of Public Health, Qatar University, Doha, Qatar

${ }^{4}$ Department of Primary Health Care \& General Practice, University of Otago,

Wellington, New Zealand

${ }^{5}$ Centre for Biomedical Ethics, National University of Singapore, Singapore

${ }^{6}$ School of Public Health and Community Medicine, University of New South Wales,

Sydney, New South Wales, Australia

\section{Twitter Lily 0'Hara @lilyohara}

Contributors $L G$ conceived the initial idea. A meeting was held via zoom with $L G$, $\mathrm{JC}, \mathrm{MH}, \mathrm{LO}, \mathrm{AB}$ and $\mathrm{AH}$ to substantially contribute to the design and scope of the review, to agree registration of the review protocol and roles in the review to be undertaken. $L G$ and JC prepared the initial outline draft manuscript. LG, JC, MH, LO, $A B$ and $A H$ contributed equally to drafting, critically revising the detailed protocol manuscript, approving all versions of the submitted protocol manuscript and are accountable for all aspects of the work.

Funding LG received a publishing award from the University of Otago, Wellington for open access publication fees.

Competing interests None declared.

Patient consent for publication Not required.

Provenance and peer review Not commissioned; externally peer reviewed.

Supplemental material This content has been supplied by the author(s). It has not been vetted by BMJ Publishing Group Limited (BMJ) and may not have been peer-reviewed. Any opinions or recommendations discussed are solely those of the author(s) and are not endorsed by BMJ. BMJ disclaims all liability and responsibility arising from any reliance placed on the content. Where the content includes any translated material, BMJ does not warrant the accuracy and reliability of the translations (including but not limited to local regulations, clinical guidelines, terminology, drug names and drug dosages), and is not responsible for any error and/or omissions arising from translation and adaptation or otherwise.

Open access This is an open access article distributed in accordance with the Creative Commons Attribution Non Commercial (CC BY-NC 4.0) license, which permits others to distribute, remix, adapt, build upon this work non-commercially, and license their derivative works on different terms, provided the original work is properly cited, appropriate credit is given, any changes made indicated, and the use is non-commercial. See: http://creativecommons.org/licenses/by-nc/4.0/.

\section{ORCID iD}

Lesley Gray http://orcid.org/0000-0001-6414-3236

\section{REFERENCES}

1 Chen N, Zhou M, Dong X, et al. Epidemiological and clinical characteristics of 99 cases of 2019 novel coronavirus pneumonia in Wuhan, China: a descriptive study. The Lancet 2020;395:507-13.

2 Huang C, Wang Y, Li X, et al. Clinical features of patients infected with 2019 novel coronavirus in Wuhan, China. The Lancet 2020;395:497-506.

3 Zhang JJY, Lee KS, Ang LW, et al. Risk factors for severe disease and efficacy of treatment in patients infected with COVID-19: a systematic review, meta-analysis, and meta-regression analysis. Clin Infect Dis 2020;71:2199-206.

4 Yang J, Zheng Y, Gou X, et al. Prevalence of comorbidities in the novel Wuhan coronavirus (COVID-19) infection: a systematic review and meta-analysis. Int $J$ Infect Dis 2020;10.

5 Khalatbari-Soltani S, Cumming RG, Delpierre C, et al. Importance of collecting data on socioeconomic determinants from the early stage of the COVID-19 outbreak onwards. J Epidemiol Community Heal 2020;74:620-3.

6 TT L, Andreadakis Z, Kumar A, et al. The COVID-19 vaccine development landscape. Nat Rev Drug Discov 2020;19:305-6.

7 Badgujar KC, Badgujar VC, Badgujar SB. Vaccine development against coronavirus (2003 to present): an overview, recent advances, current scenario, opportunities and challenges. Diabetes Metab Syndr 2020;14:1361-76.

8 Federal Drug Administration. Vaccines and related biological products Advisory Committee. [Internet], 2020. Available: https:// www.fda.gov/advisory-committees/advisory-committee-calendar/ vaccines-and-related-biological-products-advisory-committeedecember-17-2020-meeting-announcement\#event-materials

9 Burton DR, Walker LM. Rational vaccine design in the time of COVID-19. Cell Host Microbe 2020;27:695-8.

10 Bennet BM, Wolf $\mathrm{J}$, Laureano R, et al. Review of current vaccine development strategies to prevent coronavirus disease 2019 (COVID-19). Toxicol Pathol 2020;0192623320959090.

11 Smith DD, Pippen JL, Adesomo AA, et al. Exclusion of pregnant women from clinical trials during the coronavirus disease 2019 pandemic: a review of international registries. Am J Perinatol 2020;37:792.

12 Bernard MA, Clayton JA, Lauer MS. Inclusion across the lifespan: $\mathrm{NIH}$ policy for clinical research. Jama 2018;320:1535-6.

13 Pestine E, Stokes A, Trinquart L. Representation of obese participants in obesity-related cancer randomized trials. Annals of Oncology 2018;29:1582-7.

14 Vries ST, Denig P, Ekhart C, et al. Sex differences in adverse drug reactions reported to the National pharmacovigilance centre in the Netherlands: an explorative observational study. Br J Clin Pharmacol 2019;85:1507-15.

15 Omer S, Faden R, Kochhar S, et al. Who SAGE roadmap for prioritizing uses of COVID-19 vaccines in the context of limited supply. World Heal Organ, 2020.

16 Zimmermann $\mathrm{P}$, Curtis N. Factors that influence the immune response to vaccination. Clin Microbiol Rev 2019;32.

17 Painter SD, Ovsyannikova IG, Poland GA. The weight of obesity on the human immune response to vaccination. Vaccine 2015;33:4422-9.

18 Townsend MJ, Kyle TK, Stanford FC. COVID-19 Vaccination and Obesity: Optimism and Challenges.". Obesity 2021;27:634-5.

19 Federal Drug Administration. Vaccines and related biological products Advisory Committee Meeting February 26, 2021 FDA Briefing Document Janssen Ad26.COV2.S Vaccine for the Prevention of COVID-19. [Internet], 2021. Available: https://www.fda.gov/media/ 146217/download

20 Garritty C, Gartlehner G, Kamel C, et al. Cochrane rapid reviews interim guidance from the Cochrane rapid reviews methods group, 2020.

21 Moher D, Stewart L, Shekelle P. All in the family: systematic reviews, rapid reviews, scoping reviews, realist reviews, and more. Syst Rev 2015;4:183

22 Liberati A, Altman DG, Tetzlaff $\mathrm{J}$, et al. The PRISMA statement for reporting systematic reviews and meta-analyses of studies that evaluate health care interventions: explanation and elaboration. $J$ Clin Epidemiol 2009;62:e1-34.

23 Moher D, Shamseer L, Clarke M, et al. Preferred reporting items for systematic review and meta-analysis protocols (PRISMA-P) 2015 statement. Syst Rev 2015;4:1.

24 London School of Hygiene and Tropical Medicine. VaC COVID-19 vaccine tracker [Internet], 2020. Available: https://vac-Ishtm. shinyapps.io/ncov_vaccine_landscape/\%0A

25 ISRCTN Register. ISRCTN Registery [Internet]. Available: http://www. isrctn.com/search

26 Ouzzani M, Hammady H, Fedorowicz Z, et al. Rayyan-a web and mobile APP for systematic reviews. Syst Rev 2016;5:210. 
27 Elmagarmid A, Fedorowicz Z, Hammady H. Rayyan: a systematic reviews web APP for exploring and filtering searches for eligible studies for Cochrane reviews. in: Abstracts of the 22nd Cochrane Colloquium. John Wiley \& Sons, 2014: 9.
28 Sterne JAC, Savović J, Page MJ, et al. Rob 2: a revised tool for assessing risk of bias in randomised trials. BMJ 2019;2:14898.

29 Higgins JPT, Sterne JAC, Savovic J, et al. A revised tool for assessing risk of bias in randomized trials. Cochrane database Syst Rev 2016;10:29-31. 